CASE REPORT

\title{
Gastric neuroendocrine carcinoma arising from heterotopic pancreatic tissue
}

\section{R Chetty, I Weinreb}

An 85 year old man presented with symptoms of dyspepsia and increase in stool frequency of two months duration. Upper endoscopy revealed an ulcer and the biopsy was interpreted as carcinoma with endocrine features. A formal distal gastrectomy was planned, but intraoperatively, because of the patient's condition, a wedge resection was performed. Histology revealed a neuroendocrine tumour (grade 1), located mainly in the submucosa, which had caused mucosal attenuation and ulceration. Associated with the tumour and at its periphery was heterotopic pancreatic tissue composed of acini, ducts, and endocrine cells. The tumour was strongly positive for chromogranin and focally for synaptophysin. There was no associated chronic atrophic gastritis or $G$ cell hyperplasia. A discrete focus of high grade neuroendocrine carcinoma (grade 3) within the typical (grade 1) neuroendocrine tumour was also present. This case illustrates a grade 1 neuroendocrine carcinoma, with a small grade 3 focus, arising within or intimately associated with heterotopic pancreatic tissue in the stomach. Although the two entities may be separate, their close topographical association favours the possibility of neuroendocrine carcinoma arising from the heterotopic pancreas. Pathologists should be aware of the occurrence of pancreatic heterotopia in the stomach and the association of carcinoma with it.

$\mathrm{P}$ ancreatic heterotopia has been defined as "the occurrence of pancreatic tissue in aberrant anatomic sites that lack vascular, neural and anatomic continuity with the pancreas". ${ }^{1}$ In general, pancreatic heterotopia is rare and postmortem frequency rates range from $0.6 \%$ to $13.7 \% .^{2}$ It is usually asymptomatic and the common gastrointestinal sites of occurrence include the stomach, duodenum, jejunum, Meckel's diverticulum, ${ }^{13}$ gallbladder, ${ }^{4}$ and oesophagus. ${ }^{5}$ If symptomatic, abdominal pain, melaena, anaemia, obstruction, and cystic change have all been documented. ${ }^{6}$ Indeed, even carcinomas arising within heterotopic pancreatic tissue have been described..$^{7-10}$ To the best of our knowledge, a case of neuroendocrine carcinoma arising in gastric heterotopic pancreas has not been described previously.

"Pancreatic heterotopia is rare and postmortem frequency rates range from $0.6 \%$ to $13.7 \%$ "

\section{CASE REPORT}

The patient was an 85 year old man with a past medical history of cardiac arrhythmias, for which he had a pacemaker, and hypertension. He presented with a two month history of dyspepsia and an increase in stool frequency. He did not have diarrhoea, vomiting, nausea, blood per rectum, or melaena. He did not have dysphagia, change in appetite, or weight loss.

Upon presentation, he had a normal physical examination, including his abdominal examination. Specifically, there was no abdominal tenderness, masses, or organomegaly. Digital rectal examination was normal. However, he did have an incidental inguinal hernia. Occult blood was present in the stool examination of the patient. Computerised tomography scans and magnetic resonance imaging of the chest, abdomen, and pelvis revealed no lesions other than in the stomach.

The patient was booked for upper and lower endoscopy. His colonoscopy showed a single hyperplastic polyp and sigmoid diverticulosis. His upper endoscopy showed a large gastric antral ulcer. This was biopsied and the pathology suggested a carcinoma with endocrine features.

He was subsequently booked for a distal gastrectomy. His intraoperative exploration did not reveal liver metastases, peritoneal carcinomatosis, or lymphadenopathy. The lesion was palpated along the posterior aspect of the stomach, within the lesser sac. It was noted to be very mobile and did not have the consistency of an adenocarcinoma. The pancreas was not noted to contain tumour. Given these findings and the patient's age, it was decided that a simple wedge resection would suffice instead of a formal distal gastrectomy. The patient remains well one month after the procedure.

\section{MATERIALS AND METHODS}

Representative sections from the surgical specimen, which was fixed in $10 \%$ neutral buffered formalin, were processed in a routine fashion. In addition to haematoxylin and eosin stains, immunohistochemistry was performed on the formalin fixed, paraffin wax embedded tissue using the liquid streptavidin-biotin complex immunoperoxidase method, with diaminobenzidine as chromogen. Immunohistochemistry was performed with a panel of immunohistochemical markers.

\section{RESULTS}

\section{Gross examination}

The surgical specimen was received in three pieces, the largest measuring $6.5 \times 1.5 \times 1.0 \mathrm{~cm}$. The mucosal aspect appeared slightly protuberant and showed a haemorrhagic focus measuring $1.0 \times 0.7 \mathrm{~cm}$, with the remainder of the mucosa appearing normal. The serosal surface was normal. On sectioning the gastric wall, a well circumscribed, pale submucosal mass was noted, underlying the haemorrhagic focus. This mass measured $1.7 \times 1.7 \times 0.9 \mathrm{~cm}$, and it had a uniform cut surface with no areas of haemorrhage or necrosis. The mass was submitted in toto for histological assessment. 


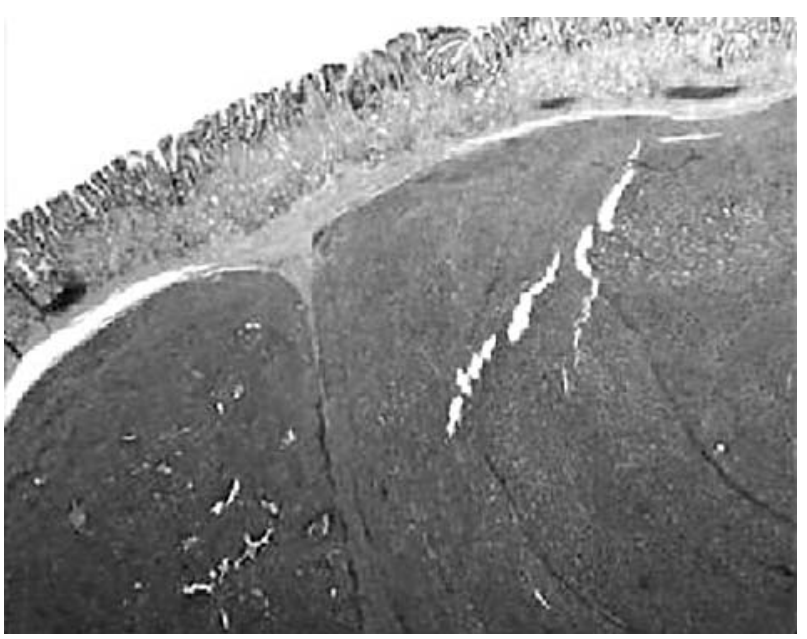

Figure 1 Low power image showing a submucosal tumour with stretched overlying mucosa.

\section{Histological examination}

A well demarcated, circumscribed submucosal tumour was identified under low power magnification. The overlying muscularis mucosae was largely intact (fig 1) except for a focal area where it was breached by the tumour. Here, small foci of the tumour lay within the lamina propria. The mucosal surface showed focal ulceration with an acute inflammatory exudate. The deep aspect of the tumour was well confined by the muscularis propria, which was not invaded or breached. At the periphery of the tumour, adjacent to the muscularis propria, was a discrete focus of pancreatic tissue composed mainly of ducts (fig 2). Very occasional acinar cells were evident and a focus of normal appearing islet cells was noted intimately associated with the neuroendocrine tumour, which appeared to be arising from these cells. The entire heterotopic focus was within the tumour mass and confined by the muscularis propria. The tumour was arranged in nests or packets surrounded by delicate capillaries, in addition to trabeculae, pseudo-papillary and pseudo-acinar patterns. Individual tumour cells were uniform with ample eosinophilic cytoplasm, and round to oval nuclei containing dispersed, salt and pepper chromatin (fig 3). There was minimal pleomorphism, and in the most mitotically active areas the mitotic count was three for each 10 high power

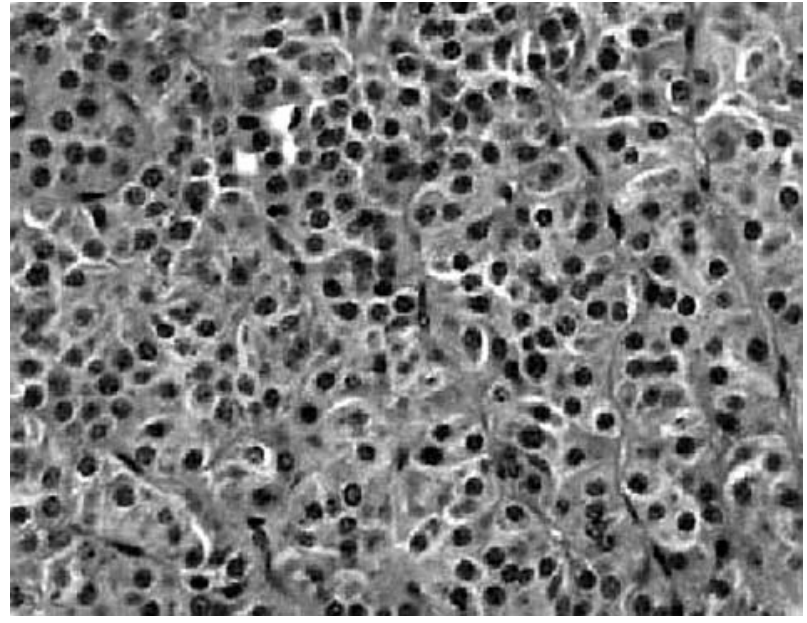

Figure 3 Typical endocrine tumour composed of uniform cells arranged in nests and trabeculae.

fields. Necrosis or areas of haemorrhage were not present. These morphological features were typical of a well differentiated gastric neuroendocrine carcinoma (grade 1). A discrete microscopic hypercellular focus at the periphery of the tumour, separate from the heterotopic pancreas, was also present (fig 4). This focus had a more trabecular pattern, with occasional pseudo-acinar areas, and was sharply demarcated from the typical neuroendocrine component. The cells were larger, elongated to spindle shaped, with vesicular nuclei, small but distinct nucleoli, and easily identifiable mitoses. This was construed as a focus of high grade neuroendocrine carcinoma (grade 3). Importantly, the overlying and adjacent gastric epithelium did not show evidence of chronic atrophic gastritis or endocrine cell hyperplasia. This last point was confirmed by the absence of $\mathrm{G}$ cell hyperplasia on gastrin immunostaining.

The typical neuroendocrine areas were strongly immunoreactive for chromogranin and focally for synaptophysin, $\mathrm{CD} 56$, and AEl/3. The high grade area showed a similar immunoprofile. The ducts in the heterotopic pancreatic tissue stained with epithelial markers, whereas the non-neoplastic endocrine cells were positive for chromogranin and focally positive for synaptophysin. The MIB-1 labelling index in both the classic grade 1 areas and the grade 3 focus was $5-10 \%$.

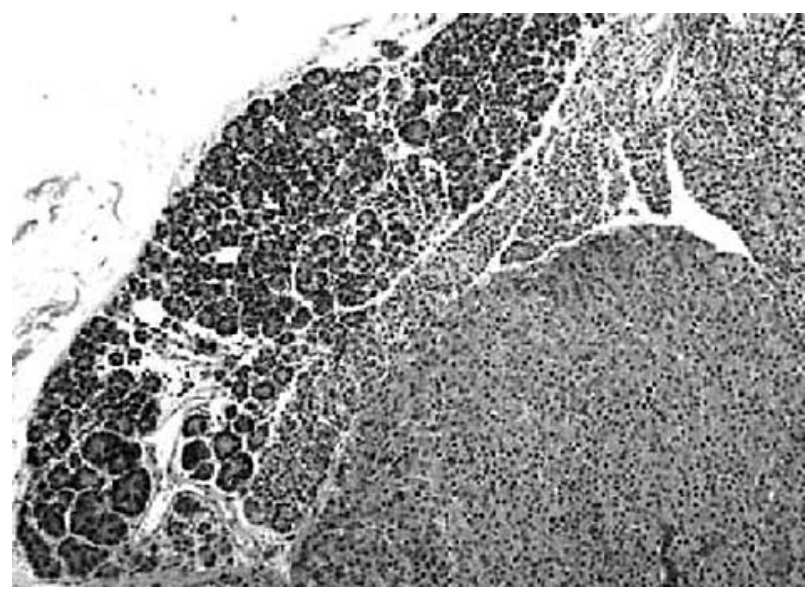

Figure 2 At one edge of the tumour clearly defined pancreatic tissue containing ducts, acini, and endocrine cells was identified. There was merging of the heterotopic pancreas with the neuroendocrine tumour.

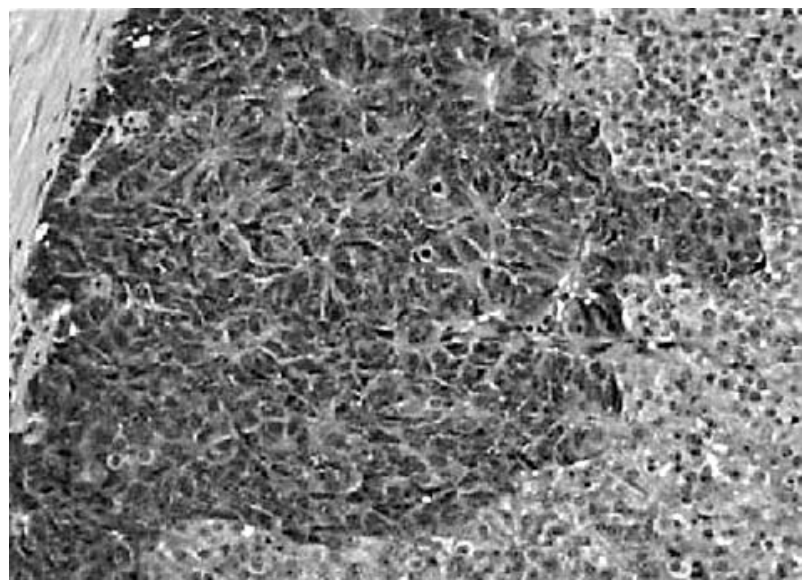

Figure 4 A focus of high grade (grade 3) neuroendocrine carcinoma with larger, hyperchromatic cells showing several mitoses. 


\section{Take home messages}

- We present the case of an 85 year old man who had a grade 1 neuroendocrine carcinoma, with a small grade 3 focus, arising within or intimately associated with heterotopic pancreatic tissue in the stomach

- Although the two entities may be separate, their close topographical association favours the possibility of neuroendocrine carcinoma arising from the heterotopic pancreas

- Practising pathologists should be aware of the occurrence of pancreatic heterotopia in the stomach and the association of carcinoma with it

The surrounding non-neoplastic tissue showed a labelling index of less than $1 \%$. All other markers were negative.

\section{DISCUSSION}

Several theories have been advanced to explain the pathogenesis and occurrence of pancreatic heterotopia. The most tenable and plausible implicates migration and/or rests of branching pancreatic tissue buds from the developing pancreas during embryogenesis. This theory is most likely when one considers gastrointestinal pancreatic heterotopia; however, pancreatic tissue occurring in more exotic, anatomically remote sites, such as the thyroid gland or fallopian tube, may have a different pathogenesis. The possibility of origin from teratomas has been advanced in these instances.

Heterotopic pancreas in the stomach is usually located within $5 \mathrm{~cm}$ of the pylorus, may occur on either anterior or posterior gastric walls, and is more common along the greater curve. ${ }^{11}$ Most cases are located within the submucosa, but intramuscular and subserosal localisation has also been encountered. ${ }^{1}$ The submucosal lesions may be sessile, polypoid, or nipple-like, where the duct associated with the heterotopic pancreas may communicate with the gastric lumen. ${ }^{1}$

Gaspar-Fuentes and colleagues modified von Heinrich's classification of pancreatic heterotopia. ${ }^{12}{ }^{13}$ Type I was total pancreatic heterotopia with all pancreatic cell types present, type II is composed of pancreatic ducts only (the so called canalicular variety), type III comprises acinar tissue only (exocrine pancreas), and type IV is made up of islet cells only (endocrine pancreas). The histological classification of the heterotopic pancreas in our case is type I because all elements were present, although the ductal component dominated.

As mentioned earlier, gastric heterotopic pancreas is most frequently asymptomatic, but when symptoms do arise they do so because of gastric mucosal erosion, so that abdominal pain, melaena, anaemia, and nausea are the usual symptoms. Unusually, obstruction of the pyloric outflow tract and mucous retention have been described.

\section{"The independent existence of two distinct pathologies- pancreatic heterotopia and neuroendocrine carcinoma- cannot entirely be excluded"}

The risk of malignancy arising in heterotopic pancreas is exceedingly rare, but several documented cases have appeared in the literature. ${ }^{814-18}$ Guillou and colleagues suggested that three criteria had to be fulfilled before a diagnosis of adenocarcinoma arising within the setting of heterotopic pancreas could be made. ${ }^{7}$ First, they stated that the tumour must be within or near the heterotopic pancreas; secondly, a transition between the pancreatic tissue and the tumour should be established (they alluded to dysplastic ducts); and thirdly, the non-neoplastic pancreatic tissue should show well developed ducts and acini. However, the third criterion does not necessarily apply, given the suggested histological classification of Gaspar-Fuentes et al. ${ }^{12}$ In the case described here, all three criteria are met, although endocrine cell dysplasia is not strictly applicable in this particular context. It is felt that our case illustrates a neuroendocrine carcinoma (grade I) arising within the context of heterotopic pancreas in the stomach, an occurrence not described previously. A less likely scenario is a metastatic neuroendocrine carcinoma to the stomach or a primary gastric neuroendocrine carcinoma with unrelated pancreatic heterotopia of the stomach, although the precise anatomical colocalisation of the two lesions argues against either of these possibilities. Furthermore, the neuroendocrine carcinoma appeared to merge or show transition with the heterotopic pancreatic tissue. Primary neuroendocrine carcinomas of the stomach are associated with G cell hyperplasia and chronic atrophic gastritis in most cases. These last two features were not present in our case. Metastatic neuroendocrine carcinoma is not likely in view of the absence of a lesion in other abdominal sites, the pelvis, and the lungs. However, the independent existence of two distinct pathologiespancreatic heterotopia and neuroendocrine carcinomacannot entirely be excluded.

Of interest is the focus of high grade (grade III) neuroendocrine carcinoma within an otherwise low grade tumour. Although not completely unexpected (given the morphological spectrum of grade I through to grade III that is described in neuroendocrine carcinoma), it is felt that the overall behaviour of the tumour may be influenced by the high grade focus.

\section{Authors' affiliations \\ R Chetty, I Weinreb, Department of Pathology, University Health Network and University of Toronto, Toronto, ON M5G 2M9, Canada}

Correspondence to: Dr R Chetty, University Health Network, Princess Margaret Hospital, 610 University Avenue, Fourth Floor, Suite 302, Room 312, Toronto, ON M5G 2M9, Canada; runjan.chetty@uhn.on.ca

Accepted for publication 15 October 2003

\section{REFERENCES}

1 Lack EE. Congenital and developmental abnormalities of the pancreas. In: Pathology of the pancreas, gallbladder, extrahepatic biliary, and ampullary region, 1st ed. New York: Oxford University Press Inc, 2003:44-62.

2 Dolan RV, ReMine WH, Dockerty MB. The fate of heterotopic pancreatic tissue. Arch Surg 1974;109:762-5.

3 Solcia E, Capella C, Kloppel G. Tumour-like lesions of the exocrine pancreas. In: Tumors of the pancreas. Atlas of tumor pathology, 3rd series, fascicle 20 Washington, DC: Armed Forces Institute of Pathology, 1997:231-2.

4 Bhana B, Chetty R. Heterotopic pancreas - an unusual cause of cholecystitis. S Afr J Surg 1999;37:105-7.

5 Razi MD. Ectopic pancreatic tissue of esophagus with massive upper gastrointestinal bleeding. Arch Surg 1966;37:105-7.

6 Bethel CAl, Luquette MH, Besner GE. Cystic degeneration of heterotopic pancreas. Pediatr Surg Int 1998;13:428-30.

7 Guillou L, Nordback P, Gerber C, et al. Ductal adenocarcinoma arising in a heterotopic pancreas situated in a hiatal hernia. Arch Pathol Lab Med 1994;118:568-71.

8 Hideki U, Ryuichi $\mathrm{D}$, Koichi $\mathrm{H}$, et al. Carcinoma arising from ectopic pancreas in the stomach. Endosonographic detection of malignant change. J Clin Ultrasound 1998;26:265-8.

9 Makhlouf HR, Almeida JL, Sobin LH. Carcinoma in jejunal pancreatic heterotopia. Arch Pathol Lab Med 1999;123:707-11.

10 Zak FG. Aberrant pancreatic carcinoma in jejunal diverticulum. Gastroenterology 1956;30:529-34.

11 Martinez NS, Morlock CG, Dockerty MB, et al. Heterotopic pancreatic tissue involving the stomach. Am Surg 1958;147:1-12.

12 Gaspar-Fuentes A, Campos Tarrech JM, Fernandez Burgui J, et al. Ectopias pancreaticas. Rev Esp Enferm Apar Dig 1973;39:255-68.

13 Hammock L, Jorda M. Gastric endocrine pancreatic heterotopia. Report of a case with histologic and immunohistochemical findings and review of the literature. Arch Pathol Lab Med 2002;126:464-7. 
14 Goldfarb WB, Benett D, Monafo W. Carcinoma in heterotopic gastric pancreas. Ann Surg 1963;158:56-8.

15 Hickman DM, Frey CF, Carson JW. Adenocarcinoma arising in gastric heterotopic pancreas. West Med J 1981;135:57-62.

16 Jeong HY, Yang HW, Seo SW, et al. Adenocarcinoma arising from an ectopic pancreas in the stomach. Endoscopy 2002;34:1014-17.
17 Osanai M, Miyokawa N, Tamaki T, et al. Adenocarcinoma arising in gastric heterotopic pancreas: clinicopathological and immunohistochemical study with genetic analysis of a case. Pathol Int 2001;51:549-54.

18 Tamimura A, Yamamoto H, Shibata $\mathrm{H}$, et al. Carcinoma in heterotopic gastric pancreas. Acta Pathol Jpn 1979;29:251-7. 